Article

\title{
Light-Irradiation Wavelength and Intensity Changes Influence Aflatoxin Synthesis in Fungi
}

\section{Tadahiro Suzuki}

Division of Food Biotechnology, Food Research Institute, NARO, 2-1-12 Kannon-dai, Tsukuba, Ibaraki 305-8642, Japan; suzut@affrc.go.jp; Tel.: +81-29-838-8063

Received: 8 December 2017; Accepted: 3 January 2018; Published: 5 January 2018

\begin{abstract}
Fungi respond to light irradiation by forming conidia and occasionally synthesizing mycotoxins. Several light wavelengths, such as blue and red, affect the latter. However, the relationship between light irradiation and mycotoxin synthesis varies depending on the fungal species or strain. This study focused on aflatoxin (AF), which is a mycotoxin, and the types of light irradiation that increase AF synthesis. Light-irradiation tests using the visible region indicated that blue wavelengths in the lower $500 \mathrm{~nm}$ region promoted AF synthesis. In contrast, red wavelengths of $660 \mathrm{~nm}$ resulted in limited significant changes compared with dark conditions. Irradiation tests with different intensity levels indicated that a low light intensity increased AF synthesis. For one fungal strain, light irradiation decreased the AF synthesis under all wavelength conditions. However, the decrease was mitigated by $525 \mathrm{~nm}$ low intensity irradiation. Thus, blue-green low intensity irradiation may increase AF synthesis in fungi.
\end{abstract}

Keywords: light wavelength; intensity; aflatoxin; mycotoxin

Key Contribution:This study represented that differences of light irradiation setting through changes of wavelength and intensity influence aflatoxin synthesis. Blue-green low intensity irradiation was the most effective condition for the aflatoxins synthesis.

\section{Introduction}

Fungi have various effects on humans, animals, and plants. Some fungi are used in fermentation, brewing, and vinification. Additionally, a number of antibiotic agents have been discovered from fungi. However, fungi also infect humans, animals and plants, causing various diseases and resulting in illness and economic losses [1-3]. It is difficult to regulate individual fungi because they are distributed across a range of conditions, although fungi used for fermentation are well controlled in limited artificial environments. Thus, identifying conditions that decrease contamination will increase crop yields and reduce the harm to animals and humans. Additionally, developing new techniques to control fungal species may lead to the development of faster and more effective food-processing methods.

The sensitivity of Chlamydomonas reinhardtii to some trichothecene mycotoxins produced by Fusarium species is influenced by changes in light intensity [4]. This algal species has general plant mechanisms, such a light-dependency, that are also observed between fungi and host plants. Fungi respond to light irradiation by changing physiologically. For instance, blue-light irradiation influences conidiation in Neurospora crassa, Bipolaris oryzae, and Trichoderma atroviride [5-9], and also influences the circadian rhythms of $N$. crassa [10]. B. oryzae has blue-light receptors named BLR1 and BLR2, and N. crassa has homologous receptors named WC-1 and WC-2. Such receptors are also conserved in Aspergillus nidulans, Aspergillus fumigatus and Aspergillus oryzae [11-14]. In addition, species with red-light receptors have been reported [11,13]. Therefore, it is highly possible that the blue-light response system is conserved among various fungal strains. This system activates conidial formation, and its receptors act as transcription factors [14]. Blue light also inhibits mycelial 
growth. A. oryzae forms conidia in response to white-light irradiation and is inhibited by red-light irradiation [14]. However, Schmidt-Heydt et al. [15] reported that blue-light irradiation inhibits the conidial formation of some Aspergillus strains. Additionally, blue-light irradiation inhibits the mycelial growth of Aspergillus carbonarius and Aspergillus westerdijkiae [16]. Thus, light irradiation influences fungal metabolism but the effects are complex.

Light irradiation affects not only primary metabolism but also secondary metabolism. Indeed, A. carbonarius and $A$. westerdijkiae, which produce the mycotoxin ochratoxin A (OTA), are affected by light irradiation, and OTA synthesis is changed [16]. OTA synthesis is increased by red-light irradiation in an A. carbonarius strain, but is decreased in an A. westerdijkiae strain. However, the OTA synthesis of A. westerdijkiae is also decreased by continuous daylight irradiation, which has a yellow to orange peak wavelength. Thus, interpreting the results is not simple. Aspergillus parasiticus BFE96p decreases the synthesis of the mycotoxin aflatoxin (AF) under both blue- and red-light conditions [15]. Additionally, Fusarium graminearum BFE1006 and Penicillium expansum BFE189 increase deoxynivalenol and citrinin, which are mycotoxins, during blue-light irradiation, respectively. Thus, the relationship between light irradiation and physiological mechanisms is complicated because the testing conditions and fungal strains used are varied. Therefore, unified testing conditions are required to interpret the influence of light irradiation.

Here, specific wavelength settings and four fungal strains were used, and the effects on one metabolite were assessed. AF was selected as the target metabolite because AF contamination is a major risk [17-19] and information on its regulatory mechanism is urgently required.

\section{Results}

\subsection{Visible Changes of Plate Cultures Caused by Conidiation and Fluorescence}

To observe the influence of light irradiation at wavelengths at both ends of the visible region, $401 \mathrm{~nm}$ and $470 \mathrm{~nm}$ blue light, and $660 \mathrm{~nm}$ and $720 \mathrm{~nm}$ red light, were used to independently irradiate fungal strains on culture plates. White light, which has blue and yellow peaks (Figure S1), was also used. To establish a uniform environment for different light radiation levels, the photon flux density (PFD) was set to $4 \mu \mathrm{mol} \cdot \mathrm{m}^{-2} \cdot \mathrm{s}^{-1}$ under all light conditions.

First, to confirm the effects of light on conidial formation, spores of two fungal strains, Aspergillus flavus IFM55891 and A. parasiticus NRRL2999, were spotted onto potato dextrose agar (PDA) plates and incubated for 2-3 days. To compare colony formation, samples were also grown under dark conditions as fungal controls. Dark conditions resulted in white colonies with limited conidial formation (Figure 1a). Under red-light conditions, both strains produced white colonies similar to the controls. However, when both fungal strains were grown under blue- and white-light conditions, yellow-greenish conidia formed.

Since blue- and red-light conditions resulted in different fungal colony phenotypes, their effects on AF synthesis were investigated. Irradiation conditions were set using wavelengths at both ends of the visible region (401 nm blue light and $720 \mathrm{~nm}$ red light), and the light intensity was the same as in the conidial formation test. Additionally, since light irradiation caused changes in fungal phenotypes regardless of the strain, Aspergillus bombysis MAFF111712 and Aspergillus nomius MAFF111739 were added to this test. The observational test of AF-derived fluorescence was conducted using PDA containing $\alpha$-cyclodextrin $(\alpha-C D)$ and activated carbon to increase the visibility of the fluorescence [20-22]. AF-derived fluorescence was observed from the undersides of the culture plates. The colonies of $A$. bombysis MAFF111712 and A. nomius MAFF111739 had increased fluorescence levels under $401 \mathrm{~nm}$ irradiation compared with the control dark condition (Figure 1b). The $720 \mathrm{~nm}$ irradiation condition resulted in the same level of fluorescence as the dark condition. However, $A$. flavus IFM55891 did not show any intensity-derived changes, while A. parasiticus NRRL2999 had a decreased fluorescence level. 
(a)

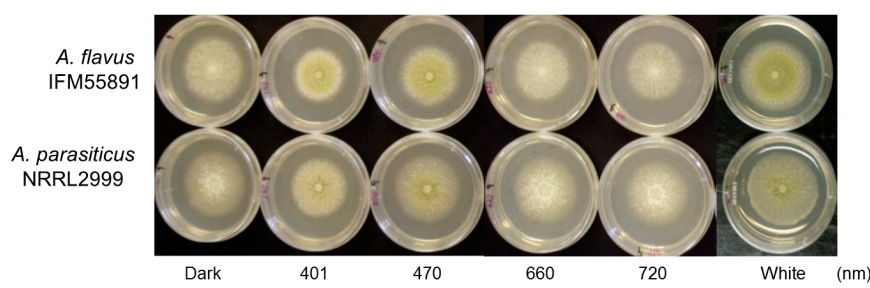

(b)
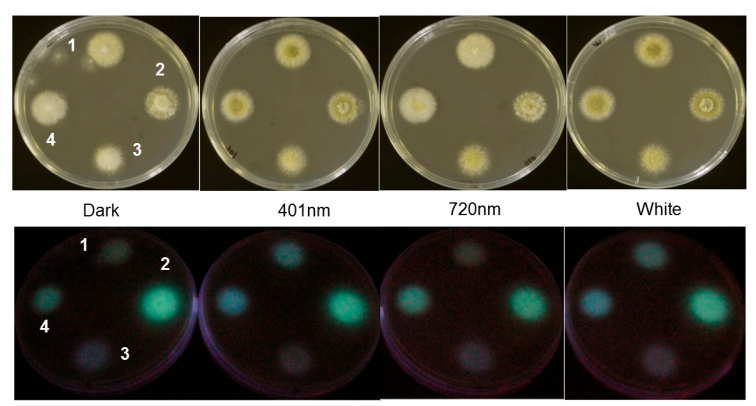

1; A. bombysis MAFF111712 2; A. parasiticus NRRL2999 $\begin{array}{ll}\text { 3: A. flavus IFM55891 } & \text { 4: } A \text {. nomius MAFF111739 }\end{array}$

Figure 1. Fungal colonies cultured under different light conditions. (a) Differences in conidial formation. Each strain was incubated on potato dextrose agar (PDA) plates under continuous light with the PFD set at $4 \mu \mathrm{mol} \cdot \mathrm{m}^{-2} \cdot \mathrm{s}^{-1}$, except in the dark controls. Yellow-green colonies were observed under $401 \mathrm{~nm}, 470 \mathrm{~nm}$ and white light, indicating conidial formation; and (b) differences in aflatoxin-derived fluorescence intensities among different light conditions. Four strains were placed on PDA plates containing $0.3 \mathrm{~g} \cdot \mathrm{L}^{-1}$ carbon and $3 \mathrm{~g} \cdot \mathrm{L}^{-1} \alpha$-cyclodextrin and incubated under different light conditions, and then the undersides of the culture plates were irradiated using $365 \mathrm{~nm}$ ultraviolet light.

Significant decreases $(p<0.05)$ in the change ratio of colony size were observed at $401 \mathrm{~nm}$ for A. flavus IFM55891 and $525 \mathrm{~nm}$ for A. bombysis MAFF111712 and A. nomius MAFF111739 compared with their respective controls under dark conditions (Figure 2a). However, continuous or wavelength-dependent changes were not observed. Measurements of AF-derived fluorescence under a wide range of wavelength conditions indicated that light irradiation increased the fluorescence intensity regardless of wavelength, except in A. parasiticus NRRL2999 (Figure 2b). In particular, during irradiation in the blue to green region (470-548 nm), the AF-derived fluorescence levels of $A$. flavus IFM55891, A. bombysis MAFF111712 and A. nomius MAFF111739 were at their highest. The intensity levels under white-light conditions were the next highest, and even irradiation using the red-light region resulted in higher fluorescence intensities than dark conditions.

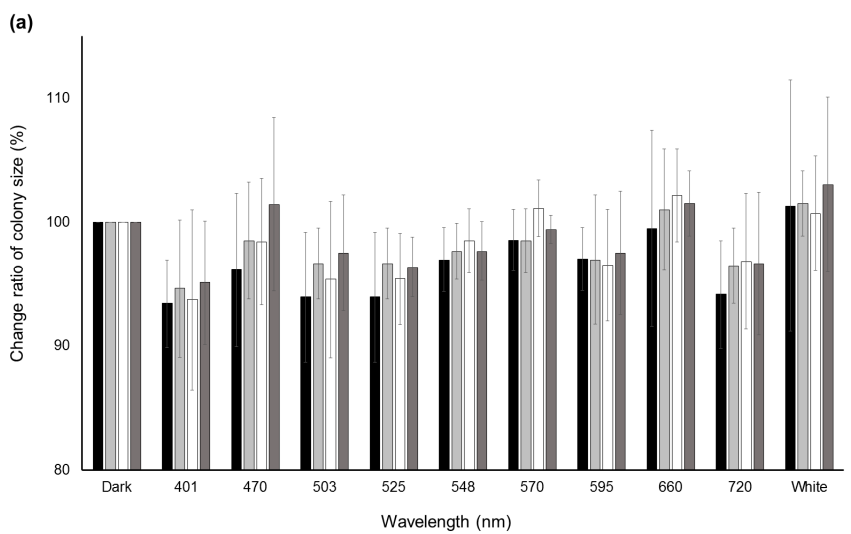

Figure 2. Cont. 
(b)

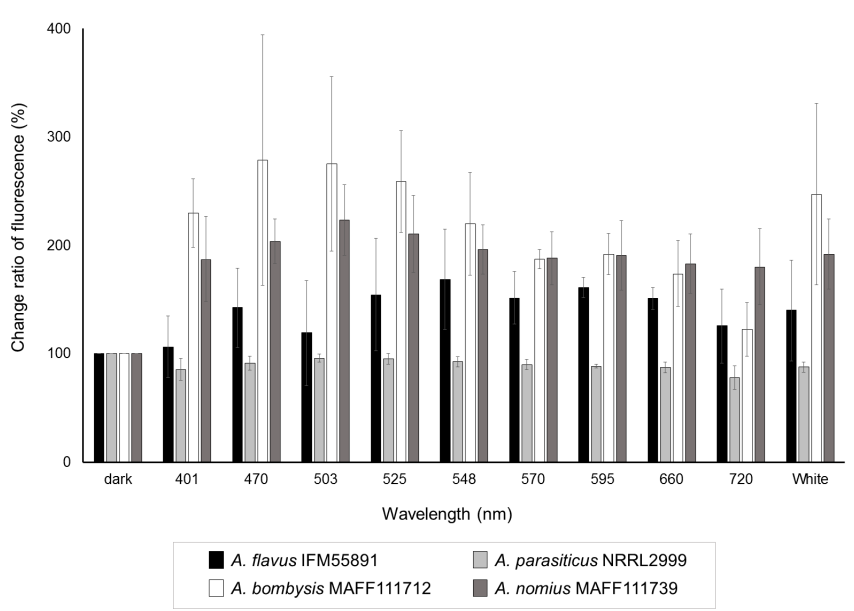

Figure 2. Change ratios of colony size and AF-derived fluorescence among different light conditions. (a) Changes in colony size after a two-day incubation under different light conditions. Colony sizes after incubation under dark conditions were standardized as the control; (b) AF-derived fluorescence obtained by UV irradiation of the undersides of the culture plates. Fluorescence intensities obtained from dark conditions were standardized as the control. Significant increases $(p<0.05)$; 548-660 nm for A. flavus IFM55891, 401 and 503-660 nm for A. bombysis MAFF111712, 470-660 nm and white for A. nomius MAFF111739. Significant decreases $(p<0.05) ; 570-660 \mathrm{~nm}$ and white for $A$. parasiticus NRRL2999. Bars indicate standard deviations; $n=3$.

\subsection{Measurement of Aflatoxins in Liquid Cultures}

The AF-derived fluorescence intensity levels of $A$. parasiticus NRRL2999 did not show significant differences in the plate test, although other fungal strains showed increased intensity levels as a result of light irradiation. Moreover, the peak radiation regions that caused the highest fluorescence intensity levels differed among the three fungal strains. Thus, the most effective wavelength for AF synthesis was not clear. To solve this problem, the AF volume was measured using a micropipette tip-based liquid culture method [23] (Figure S2). To correspond to the plate culture test, the method used liquid PD medium containing activated carbon. While adding $\alpha-C D$ to the culture plate does not increase AF synthesis in fungi, AF inclusion products increase the fluorescence intensity through an optical effect [24,25]; thus, $\alpha$-CD was not added to the liquid culture to avoid contamination with AF inclusion products. To make the liquid culture conditions close to the plate culture conditions, the incubation time was set to three days, and the same four fungal strains were used. The light conditions were also the same as for the culture plate test.

Slight decreases at $401 \mathrm{~nm}$ were observed for the fungal masses formed in each pipette tip, although wavelength-dependent changes were not observed (Figure S3). After collecting the culture medium, four types of $\mathrm{AF}\left(\mathrm{AFB}_{1}, \mathrm{AFB}_{2}, \mathrm{AFG}_{1}\right.$, and $\left.\mathrm{AFG}_{2}\right)$ were measured (Table S1). Under the single-wavelength irradiation conditions between $470 \mathrm{~nm}$ and $548 \mathrm{~nm}$, all of the fungal strains displayed maximum AF volumes. Compared with the dark conditions, A. nomius MAFF111739 had an increased AF-synthesis level at $401 \mathrm{~nm}$, while the other strains showed decreased levels.

Furthermore, AF synthesis decreased or changed insignificantly from 570 to $660 \mathrm{~nm}$, except in A. nomius MAFF111739. The amount of AF synthesis under white-light conditions was similar to that from 470 to $548 \mathrm{~nm}$. When compared with dark conditions, the AF synthesis in A. parasiticus NRRL2999 decreased, except under bluish- and white-light conditions.

The AF concentrations calculated based on the AF amounts per unit fungal dry mass also indicated that bluish irradiation resulted in maximum values, and the results corresponded to the AF synthesis trend (Figure 3). Statistical analyses were conducted to confirm how AF synthesis changed at each irradiation level (Table 1). The dark-condition samples were set as controls, and the 
significant differences at each wavelength were identified by conducting an unpaired or Welch's $t$-test. Irradiation in the lower $500 \mathrm{~nm}$ region, focused on $525 \mathrm{~nm}$, caused a significant increase in AF synthesis. The number of significant changes decreased in the higher end of the $500 \mathrm{~nm}$ region, the fewest significant changes were observed at $660 \mathrm{~nm}$. The number of significant differences under white-light conditions followed that of the lower $500 \mathrm{~nm}$ region.
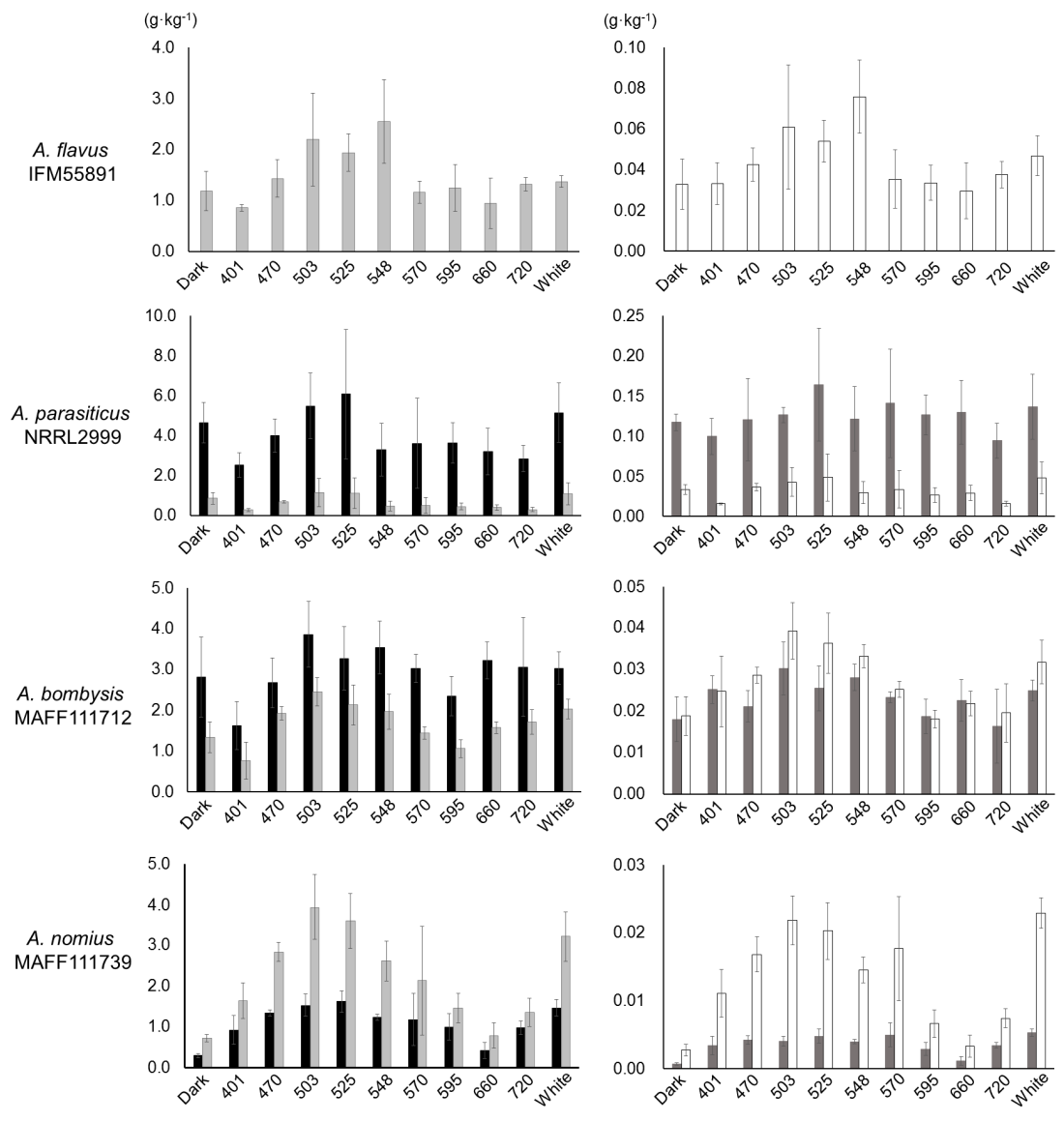

Wavelength $(\mathrm{nm})$

Wavelength $(\mathrm{nm})$

$\square \mathrm{AFB}_{1}$

$A F G_{1}$

$\square \mathrm{AFB}_{2} \quad \square \mathrm{AFG}_{2}$

Figure 3. Aflatoxin concentrations per unit fungal mass $\left(\mathrm{g} \cdot \mathrm{kg}^{-1}\right)$ obtained by integrating the fungal dry mass (Figure S3) and aflatoxin amounts (Figure S4). Bars indicate standard deviation; $n=3-6$.

Table 1. Estimation of the light-radiation region that produces the most significant increase in AF synthesis.

\begin{tabular}{ccccccccccc}
\hline & 401 & 470 & 503 & 525 & 548 & 570 & 595 & 660 & 720 & White \\
\hline $\mathrm{A}$ & $* * * *$ & $* * * *$ & $* * * *$ & $1++* *$ & $++* *$ & $* * * *$ & $* * * *$ & $* * * *$ & $* * * *$ & $* * * *$ \\
$\mathrm{~B}$ & $2-$ & $* * * *$ & $* * * *$ & $* * * *$ & $* * * *$ & $* * * *$ & $-* * *$ & $-* * *$ & -- & $* * * *$ \\
$\mathrm{C}$ & $3 * * * *$ & $*+* *$ & $++{ }^{*}+$ & $++* *$ & $*+*+$ & $*+* *$ & $* * * *$ & $* * * *$ & $* * * *$ & $++* *$ \\
$\mathrm{D}$ & ++++ & ++++ & ++++ & ++++ & ++++ & $*+*+$ & ++++ & $* * * *$ & $*+++$ & ++++
\end{tabular}

1 A plus sign indicates a significant increase $(p<0.05)$ in aflatoxin synthesis. ${ }^{2}$ A minus sign indicates a decrease $(p<0.05)$ in aflatoxin synthesis. ${ }^{3}$ Asterisk; no significant change detected. The set of plus or minus signs in each cell reflects significant changes of $\mathrm{AFB}_{1}, \mathrm{AFB}_{2}, \mathrm{AFG}_{1}$, and $\mathrm{AFG}_{2}$, respectively. A; A. flavus IFM55891, $\mathrm{B} ;$ A. parasiticus NRRL2999, C; A. bombysis MAFF111712, D; A. nomius MAFF111739. 


\subsection{Exposure to Different Irradiation Intensity Levels Results in Varied Aflatoxin Synthesis}

In this study, statistical analyses indicated that $525 \mathrm{~nm}$ irradiation induced the maximum AF synthesis level and that $660 \mathrm{~nm}$ irradiation caused few significant changes. Past studies investigating the relationship between light irradiation wavelengths and mycotoxin production have not produced unified results $[15,16]$. One explanation for these differences may be the use of different fungal strains. Additionally, differences in conditions other than wavelength, such as the irradiation intensity, might have influenced the efficiency of AF synthesis. Initially, a $4-\mu \mathrm{mol} \cdot \mathrm{m}^{-2} \cdot \mathrm{s}^{-1}$ intensity setting was used in this study, which was low compared with that used in past studies. Thus, another test was conducted using an intensity of $40 \mu \mathrm{mol} \cdot \mathrm{m}^{-2} \cdot \mathrm{s}^{-1}$ at $525 \mathrm{~nm}$ and $660 \mathrm{~nm}$, and the effects on AF synthesis were compared with those at $4 \mu \mathrm{mol} \cdot \mathrm{m}^{-2} \cdot \mathrm{s}^{-1}$.

Fungal growth levels and mycelial masses remained unchanged, except the masses of $A$. flavus IFM55891 and A. nomius MAFF111739 under dark conditions (Figure S4). For A. flavus IFM55891, AF synthesis under light-irradiation conditions was greater than under dark conditions, regardless of the irradiation intensity or wavelength (Table S2). According to calculations of the AF amounts per unit fungal dry mass, $4 \mu \mathrm{mol} \cdot \mathrm{m}^{-2} \cdot \mathrm{s}^{-1}$ at $525 \mathrm{~nm}$ conditions resulted in the highest level of AF synthesis (Figure 4). The results for A. bombysis MAFF111712 were similar. A. nomius MAFF111739 had the highest AF synthesis level under $40 \mu \mathrm{mol} \cdot \mathrm{m}^{-2} \cdot \mathrm{s}^{-1}$ at $525 \mathrm{~nm}$ conditions, although other irradiation-condition results corresponded to those of A. flavus IFM55891 and A. bombysis MAFF111712. Only A. parasiticus NRRL2999 differed from the other strains, with the highest AF synthesis occurring under dark conditions. Overall, the $4 \mu \mathrm{mol} \cdot \mathrm{m}^{-2} \cdot \mathrm{s}^{-1}$ irradiation intensity increased AF synthesis more than $40 \mu \mathrm{mol} \cdot \mathrm{m}^{-2} \cdot \mathrm{s}^{-1}$ irradiation, although there were exceptions.
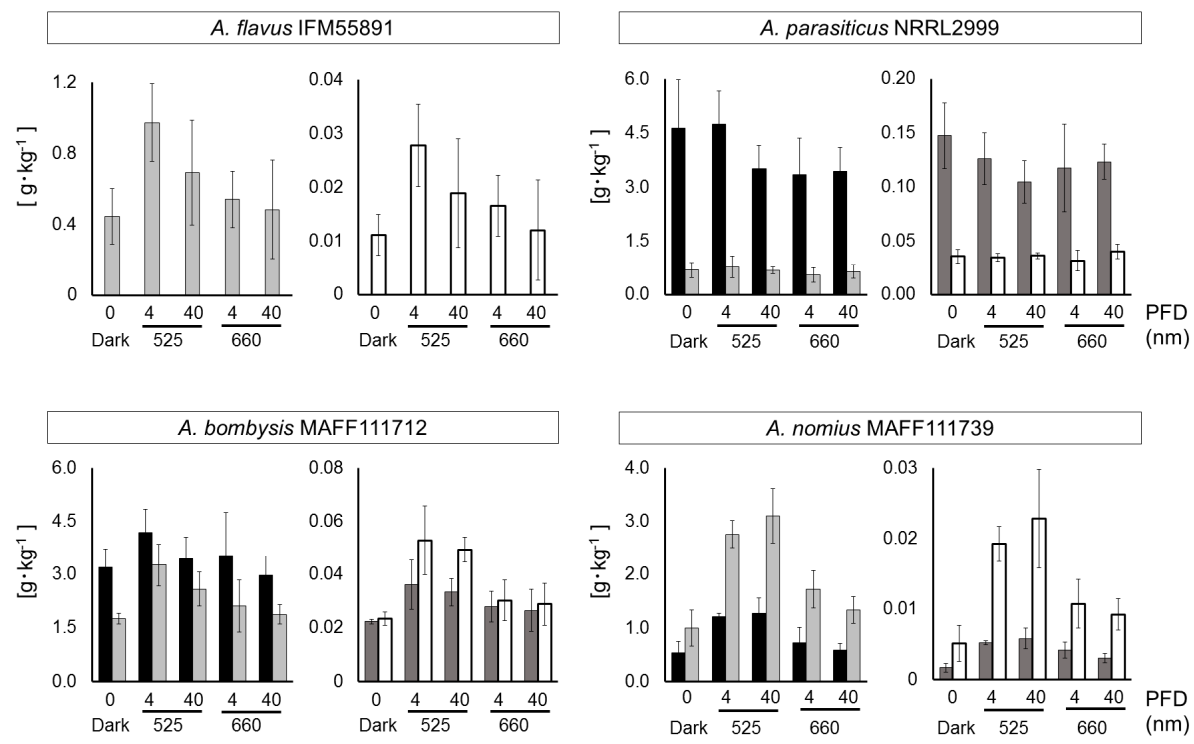

$\square \mathrm{AFB}_{1}$

$\mathrm{AFG}_{1}$

$\square \mathrm{AFB}_{2} \quad \square \mathrm{AFG}_{2}$

Figure 4. Aflatoxin concentrations per unit fungal mass $\left(\mathrm{g} \cdot \mathrm{kg}^{-1}\right)$ that were obtained by integrating the fungal dry mass (Figure S5) and aflatoxin amounts (Figure S6). Light intensities of 4 and $40 \mu \mathrm{mol} \cdot \mathrm{m}^{-2} \cdot \mathrm{s}^{-1}$ at 525 and $660 \mathrm{~nm}$ were used, except under dark conditions. Each sample was newly prepared for the comparison study. Significant increases $(p<0.05) ; 4 \mu \mathrm{mol} \cdot \mathrm{m}^{-2} \cdot \mathrm{s}^{-1}$ at $525 \mathrm{~nm}$ conditions: $\mathrm{AFB}_{1}$ and $\mathrm{AFB}_{2}$ for A. flavus IFM55891, $\mathrm{AFB}_{1}, \mathrm{AFB}_{2}$, and $\mathrm{AFG}_{2}$ for $A$. bombysis $\mathrm{MAFF} 111712$, all AFs for $A$. nomius MAFF111739; $40 \mu \mathrm{mol} \cdot \mathrm{m}^{-2} \cdot \mathrm{s}^{-1}$ at $525 \mathrm{~nm}$ conditions: $\mathrm{AFB}_{1}, \mathrm{AFB}_{2}$, and AFG2 for A. bombysis MAFF111712, all AFs for $A$. nomius MAFF111739; $4 \mu \mathrm{mol} \cdot \mathrm{m}^{-2} \cdot \mathrm{s}^{-1}$ at $660 \mathrm{~nm}$ conditions: $\mathrm{AFB}_{1}, \mathrm{AFB}_{2}$, and $\mathrm{AFG}_{2}$ for $A$. nomius MAFF111739; $40 \mu \mathrm{mol} \cdot \mathrm{m}^{-2} \cdot \mathrm{s}^{-1}$ at $660 \mathrm{~nm}$ conditions: $\mathrm{AFG}_{2}$ for A. nomius MAFF111739. Bars indicate standard deviation; $n=4$. 


\section{Discussion}

\subsection{Blue-Light Irradiation Facilitates Conidial Formation under Low-Intensity Conditions}

The relationship between light irradiation and fungi has been investigated, and the most obvious phenotypical change is in conidial formation $[14,15]$. The light-response mechanism related to conidial formation has become clear [12,26], and may be distributed throughout fungi. However, different responses to the same color of light radiation have been reported in different fungal strains $[15,16]$. Therefore, knowing the phenotypic characteristics of test strains is a preliminary requirement for light-irradiation studies. Here, the light-irradiation test indicated that blue-light conditions of $401 \mathrm{~nm}$ and $470 \mathrm{~nm}$ irradiation stimulated conidial formation, and white-light conditions also resulted in conidial formation (Figure 1a). This implies that conidial formation is not prevented under irradiation conditions by specific light wavelengths if there is a component of the blue-light region present. These trends were also seen in A. flavus IFM55891 and A. parasiticus NRRL2999, confirming that this was not a specific condition. Previous studies have presented partially corresponding and differing data compared with those of our study [14,15]. However, these studies used different strains or irradiation conditions, making direct comparisons difficult.

\subsection{Fungal Plate Cultures Undergoing Light Irradiation Provide an Improved Detection Technique for Aflatoxin Synthesis}

Since conidial formation represents a large physiological change, blue-light irradiation should affect AF synthesis. Clarifying the mechanism will be useful in regulating AF synthesis. Therefore, the relationship between light irradiation and AF synthesis was investigated. Using fungal plate cultures with light irradiation increased the observational efficiency for AF-derived fluorescence from A. bombysis MAFF111712 and A. nomius MAFF111739 under $401 \mathrm{~nm}$ irradiation conditions compared with dark conditions (Figure 1b). The fluorescence intensity levels of $A$. bombysis MAFF111712 and A. nomius MAFF111739 induced after a few days of incubation were not originally at high levels. Thus, it was expected that the blue-light irradiation would effectively increase the AF-derived fluorescence observed from the plate cultures. However, increased fluorescence intensity after blue-light irradiation was not observed for A. flavus IFM55891 or A. parasiticus NRRL2999. Thus, it is difficult to determine whether the different irradiation conditions induced the changes in fluorescence intensity levels. For white-light irradiation, both conidial formation and increased fluorescence intensity were observed, similar to the blue-light irradiation conditions. These changes correlate, on the whole, with those of the blue-light components. However, for A. nomius MAFF111739, 720 nm red-light irradiation increased the AF fluorescence despite the low level of conidial formation. In contrast, for A. flavus IFM55891 and A. parasiticus NRRL2999, $401 \mathrm{~nm}$ blue-light irradiation increased conidial formation, even though AF-derived fluorescence intensity levels were not increased. The test of specific wavelengths also showed a similar trend (Figure $2 \mathrm{~b}$ ). These different phenotypic changes among fungal strains suggest that the regulation of AF synthesis and conidial formation are separately influenced by light irradiation, although there is a commonality in that the DNA methyltransferase protein contributes to both [26]. Three strains showed fluorescence intensity maximums under blue-green-light conditions (Figure 2b). However, the irradiation wavelengths that induced the highest values were slightly misaligned and, therefore, a specific wavelength that increases AF synthesis the most in all fungal strains could not be determined. This paradoxically suggests that it is not necessarily the specific environment, including the applied single-wavelength irradiation condition that results in increased AF synthesis. The addition of general blue- or white-light irradiation to the culture conditions may help us to identify AF-synthetic fungi if the fungal strain has a low AF-synthesis level or it is hard to recognize this characteristic. 
3.3. Low-Intensity Bluish-Green-Light Irradiation Is More Effective in Increasing AF Synthesis than High-Intensity Irradiation or Other Wavelength Conditions, Although This Is Not Universal among Fungi

In this study, a liquid culture test was conducted to quantitate the levels of AF synthesis (Table S1, Figure 3). Slight changes that were hard to discriminate by direct observation using plate cultures were observed for A. flavus IFM55891 and A. parasiticus NRRL2999, and bluish-green-light irradiation composed of the lower half of the $500 \mathrm{~nm}$ region increased AF synthesis. At the same time, the AF synthesis of $A$. parasiticus NRRL2999 decreased, except under $500 \mathrm{~nm}$ irradiation. These changes in AF amounts suggested that exposure to light, except bluish-green-light irradiation, resulted in the suppression of AF synthesis. This trend was also reported in a previous test using A. parasiticus BFE96p under green-light conditions [15]. White light, which contains blue- and yellow-wavelength regions, showed the same synthesis trend as $500 \mathrm{~nm}$ irradiation. Thus, the liquid culture test supports that bluish-green- and white-light irradiation are useful for increasing or maintaining AF synthesis. In particular, $525 \mathrm{~nm}$ irradiation may be the most effective condition for AF synthesis (Table 1). However, blue-light irradiation is not always useful in increasing mycotoxin synthesis $[15,16]$. Thus, the results of this study do not entirely correspond to those of previous studies. However, different conditions were applied in each study, including light intensity, which we adjusted in this study. First, we used $4 \mu \mathrm{mol} \cdot \mathrm{m}^{-2} \cdot \mathrm{s}^{-1}$ as the low-intensity condition, which may be preferred by fungi, although other reports have used higher intensities. Then, we applied a high-intensity setting of $40 \mu \mathrm{mol} \cdot \mathrm{m}^{-2} \cdot \mathrm{s}^{-1}$, and the results were compared with those of the low-intensity condition. The low-intensity setting resulted in increased AF synthesis compared with the $40-\mu \mathrm{mol} \cdot \mathrm{m}^{-2} \cdot \mathrm{s}^{-1}$ high-intensity condition (Table S2, Figure 4). In particular, the AF synthesis of $A$. parasiticus NRRL2999 at $40 \mu \mathrm{mol} \cdot \mathrm{m}^{-2} \cdot \mathrm{s}^{-1}$ was similar to that under red-light conditions, and the synthesis at $4 \mu \mathrm{mol} \cdot \mathrm{m}^{-2} \cdot \mathrm{s}^{-1}$ was similar to that under the dark condition.

These trends were different from those of other strains. Thus, A. parasiticus NRRL2999 might have a different light-response system. A. bombysis MAFF111712 showed a relatively small change in $\mathrm{AFG}_{1}$. All of the AF fractions of $A$. parasiticus NRRL2999 and AFG 1 of A. bombysis MAFF111712, which were not greatly increased by $525 \mathrm{~nm}$ irradiation, were synthesized in large amounts under the dark condition compared with the other strains. This suggested that $525 \mathrm{~nm}$ irradiation is useful for strains that produce small amounts of AF under dark conditions. In contrast, it may have an adverse effect on strains that synthesize large amounts of AF. The fungal light-response system is regulated through a mitogen-activated pathway, which also regulates some stress responses [12]. Considering this mechanism, high amounts of AF in the culture media might act as a stressor. The relationship between the AF-synthesis mechanism and light responses may vary, and the results of this study will help to interpret how light stimuli influences AF synthesis. This study used a three-day incubation period for both plate and liquid culture tests to unify the incubation conditions and, therefore, the influence of a longer incubation period is unknown. The results with the three-day incubation period are different from those of $A$. carbonarius, in which OTA was increased by red-light irradiation [16]. In contrast, this study implies that the blue-light stimulus is related to the AF-synthesis mechanism. The red-light stimulus resulted in only small changes in AF synthesis, suggesting that the relationship between blue-light irradiation and AF synthesis is more important. However, even under red-light conditions, an increase in AF synthesis was observed, except in A. parasiticus NRRL2999. Therefore, it is unlikely that the increase is regulated by the blue-light response mechanism alone. In fact, AF synthesis in A. parasiticus BFE96p and OTA synthesis in A. westerdijkiae are repressed by both blue- and red-light irradiation $[15,16]$. However, the irradiation conditions used in those studies were not consistent. Therefore, the trends of AF and OTA synthesis might have changed owing to other irradiation conditions. Like A. parasiticus BFE96p, the AF level produced by A. parasiticus NRRL2999 at $40 \mu \mathrm{mol} \cdot \mathrm{m}^{-2} \cdot \mathrm{s}^{-1}$ decreased, whereas A. parasiticus NRRL2999 maintained its AF level at $4 \mu \mathrm{mol} \cdot \mathrm{m}^{-2} \cdot \mathrm{s}^{-1}$. This suggests that the light intensity, like the wavelength, is important. Based on the four strains used here, the low-intensity condition is more suitable for AF synthesis. 


\section{Conclusions}

The physiological behaviors of fungal strains in response to light vary depending on the light conditions or fungal strains used. Thus, a mechanistic link between mycotoxin synthesis and light irradiation cannot be presumed. However, the changes observed in our, and previous, studies suggest that light stimuli affect mycotoxin synthesis. Clarifying this situation will help develop a regulatory method to inhibit mycotoxin synthesis by influencing the physiological processes of fungi.

\section{Materials and Methods}

\subsection{Fungal Species}

Fungal strains were obtained as follows: A. flavus IFM55891 (NBRC 33021, ATC C22546) from Chiba University's Medical Mycology Research Center (Chiba, Japan), A. parasiticus NRRL2999 from Prof. Kimiko Yabe at the Faculty of Environmental and Information Science, Fukui University of Technology (Fukui, Japan), A. bombysis MAFF111712 and A. nomius MAFF111739 from the Genebank Project, National Agriculture and Food Research Organization (Ibaraki, Japan). Each sample was cultured on a PDA (Merck, Darmstadt, Germany) slant, and incubated at $28^{\circ} \mathrm{C}$ for several days. Spores were collected using $0.05 \%$ Tween 80 (ICN Biomedicals, Santa Ana, CA, USA), and stored at $4{ }^{\circ} \mathrm{C}$.

\subsection{Culture Conditions and Apparatus}

To observe conidial formation, PDA plates without any additional reagents were first prepared. To observe AF-derived fluorescence, PDA supplemented with $3 \mathrm{~g} \cdot \mathrm{L}^{-1} \alpha-\mathrm{CD}$ (Wako Pure Chemical Industries, Osaka, Japan) and $0.3 \mathrm{~g} \cdot \mathrm{L}^{-1}$ activated charcoal powder (Neutral, Wako Pure Chemical Industries) was prepared. PD broth (Merck, Darmstadt, Germany) with activated carbon and without $\alpha-C D$ was also prepared as a liquid culture solution. Then, $495 \mu \mathrm{L}$ of liquid medium was dispensed into 1-mL pipette tips, which were used as incubation apparatus (Figure S2), and the total amount was adjusted to $500 \mu \mathrm{L}$ by adding the spore solution. Each spore solution $(5 \mu \mathrm{L})$ was dispensed onto a culture plate or into the liquid culture medium. After liquid cultivation, the laboratory film (PM-996, Bemis, Neenah, WI, USA) wrapped around the top of each 1-mL tip was removed, and the tips were centrifuged at $2000 \times g$ for $10 \mathrm{~min}$. The obtained culture solutions were used to measure the AF concentrations. The mycelia with spores in the incubation tips were dried in a dehydrator (SLI-450N, TOKYO RIKAKIKAI, Tokyo, Japan) at $65^{\circ} \mathrm{C}$ overnight, and the fungal dry weight was measured by comparing the tip weight before and after incubation.

\subsection{Light Conditions and Measurement of Fluorescence Intensity Levels}

Light conditions were manually set up using shell-type light-emitting diodes on platforms (SPL-100-CC; Revox, Kanagawa, Japan). White, 401 nm, 470 nm, 503 nm, 525 nm, 548 nm, 570 nm, $595 \mathrm{~nm}, 660 \mathrm{~nm}$, and $720 \mathrm{~nm}$ diodes (Figure S1) were selected for wavelengths in the visible region. The PFD was regulated by a pulse-width modulation dimmer controller. The spectrum of light-emitting diode radiation was measured by an illuminance spectrophotometer (CL-500A; Konica Minolta, Tokyo, Japan) as irradiance $\left(\mathrm{W} / \mathrm{m}^{2}\right)$. The total spectrum from 360 to $780 \mathrm{~nm}$ was counted, and the PFD of each spectral condition was calculated using the following formula: PFD $\left(\mu \mathrm{mol} \cdot \mathrm{m}^{-2} \cdot \mathrm{s}^{-1}\right)=$ [irradiance $\left(\mathrm{W} \cdot \mathrm{m}^{-2}\right) \times$ spectrum $(\mathrm{m}) \times 10^{-9}$ ]/[Planck's constant $\left(6.626 \times 10^{-34} ; \mathrm{J} \cdot \mathrm{s}\right) \times$ speed of light $\left(2.998 \times 10^{8} ; \mathrm{m} \cdot \mathrm{s}^{-1}\right) \times$ Avogadro constant $\left.\left(6.022 \times 10^{23} ; \mathrm{mol}^{-1}\right)\right] \times 10^{6}$. Each culture sample, except under the dark condition, was continuously irradiated with 4 or $40 \mu \mathrm{mol} \cdot \mathrm{m}^{-2} \cdot \mathrm{s}^{-1}$ at different light wavelengths. The plates were incubated at $28{ }^{\circ} \mathrm{C}$ for two days, and the liquid culture media were incubated without shaking for three days. Two Aspergillus strains (A. flavus IFM55891 and A. parasiticus NRRL2999) were incubated on plain PDA plates, and four Aspergillus strains (A. flavus IFM55891, A. parasiticus NRRL2999, A. bombysis MAFFF111712 and A. nomius MAFF111739) were incubated on carbon-containing plates. As with plain PDA, the culture plates supplemented with $\alpha-C D$ and activated carbon were incubated for two days, and irradiated with UV light at $365 \mathrm{~nm}$ (UVGL-58, UVP, 
Upland, CA, USA) to confirm AF synthesis. The fluorescence signals were captured using a digital camera set to the manual white-balance setting (Caplio R3, Ricoh, Tokyo, Japan). The fluorescence intensity levels were extracted with digital image analysis software (ImageJ, version 1.40g, National Institutes of Health, Bethesda, MD, USA [27]). The changes in the fluorescence intensity levels were calculated using triplicate samples.

\subsection{High-Performance Liquid Chromatography}

Culture medium and an equivalent volume of chloroform were dispensed into new microtubes and vortexed for $10 \mathrm{~s}$. The chloroform was collected and transferred to a new microtube, and then reduced to dryness in a draft chamber at $40^{\circ} \mathrm{C}$. Subsequent procedures followed the official method [28] (in Japanese. Officially, the limitations of quantitation and detection are $1 \mu \mathrm{g} / \mathrm{kg}$ and $0.5 \mu \mathrm{g} / \mathrm{kg}$, respectively). For our instrument, the limitations of quantitation and detection were $2 \mu \mathrm{g} / \mathrm{kg}$ and $1 \mu \mathrm{g} / \mathrm{kg}$, respectively. An aliquot of trifluoroacetic acid (Wako Pure Chemical Industries, Osaka, Japan) equal to 0.1 times the volume of chloroform was added to the microtube for derivatizing $\mathrm{AFB}_{1}$ and $\mathrm{AFG}_{1}$, and the tube was vortexed for $5 \mathrm{~s}$. After incubation for more than $10 \mathrm{~min}$, a solution of acetonitrile in distilled water $(10: 90, v / v)$ was added to the microtube, with the volume equal to 0.9 times the volume of chloroform. An aliquot $(20 \mu \mathrm{L})$ of the sample solution was injected into a high-performance liquid chromatography system (SCL-10A, Shimadzu, Kyoto, Japan) with a fluorescence detector ( $\lambda_{\mathrm{Ex}}=365 \mathrm{~nm}$ and $\lambda_{\mathrm{Em}}=455 \mathrm{~nm}$; RF-535, Shimadzu, Kyoto, Japan). The mobile phase, which is a mixture of distilled water:methanol:acetonitrile $(60: 30: 10, v / v / v)$, flowed through the column (Inertsil ODS-3, column size $4.6 \times 150 \mathrm{~mm}$, particle size $5 \mu \mathrm{m}$; GL Sciences, Tokyo, Japan) at a rate of $1 \mathrm{~mL} \cdot \mathrm{min}^{-1}$ to separate AFs.

\subsection{Statistical Analyses}

Average values of colony size, fluorescence intensity, fungal weight, and AF level were calculated, and the values under the dark condition were set as the control in each comparison. All comparison conditions were analyzed using an $F$-test, and then equally distributed conditions $(p<0.05)$ were analyzed using an unpaired $t$-test. For the unequally distributed conditions, Welch's test was conducted. For the comparisons of AF concentrations, significant increases and decreases were discriminated, and indicated using plus or minus symbols, respectively. Each sample condition was prepared at least in triplicate.

Supplementary Materials: The following are available online at www.mdpi.com/2072-6651/10/1/31/s1. Figure S1: Light spectra used in this study, Figure S2: Diagrammatic illustration of the modified tip-culture method, Figure S3: Changes in fungal dry mass after a three-day incubation period in liquid culture media at the $4 \mu \mathrm{mol} \cdot \mathrm{m}^{-2} \cdot \mathrm{s}^{-1}$ setting, Figure S4: Changes in fungal dry mass after a three-day incubation period in liquid culture media, Table S1: Aflatoxin concentrations per amount $\left(\mathrm{mg} \cdot \mathrm{L}^{-1}\right)$ synthesized after a three-day incubation period in liquid culture media, Table S2: Aflatoxin concentrations per amount $\left(\mathrm{mg} \cdot \mathrm{L}^{-1}\right)$ synthesized after a three-day incubation period in liquid culture media.

Acknowledgments: The author thanks Kimiko Yabe (Faculty of Environmental and Information Science, Fukui University of Technology) for providing the fungal strains. This work was supported by JSPS KAKENHI Grant Number 16K21606. We thank Lesley Benyon, from Edanz Group (www.edanzediting.com/ac) for editing a draft of this manuscript.

Conflicts of Interest: The author declares no conflict of interest.

\section{References}

1. Wu, F. Measuring the economic impacts of Fusarium toxins in animal feeds. Anim. Feed Sci. Technol. 2007, 137, 363-374. [CrossRef]

2. Gibb, H.; Devleesschauwer, B.; Bolger, P.M.; Wu, F.; Ezendam, J.; Cliff, J.; Zeilmaker, M.; Verger, P.; Pitt, J.; Baines, J.; et al. World Health Organization estimates of the global and regional disease burden of four foodborne chemical toxins, 2010: A data synthesis. F1000Res 2015, 4, 1393. [CrossRef] [PubMed] 
3. Misihairabgwi, J.M.; Ezekiel, C.N.; Sulyok, M.; Shephard, G.S.; Krska, R. Mycotoxin contamination of foods in Southern Africa: A 10-year review (2007-2016). Crit. Rev. Food Sci. Nutr. 2017, 1-16. [CrossRef] [PubMed]

4. Suzuki, T.; Iwahashi, Y. Phytotoxicity evaluation of type B trichothecenes using a Chlamydomonas reinhardtii model system. Toxins (Basel) 2014, 6, 453-463. [CrossRef] [PubMed]

5. Kihara, J.; Moriwaki, A.; Tanaka, N.; Ueno, M.; Arase, S. Characterization of the BLR1 gene encoding a putative blue-light regulator in the phytopathogenic fungus Bipolaris oryzae. FEMS Microbiol. Lett. 2007, 266, 110-118. [CrossRef] [PubMed]

6. Moriwaki, A.; Katsube, H.; Ueno, M.; Arase, S.; Kihara, J. Cloning and characterization of the BLR2, the homologue of the blue-light regulator of Neurospora crassa WC-2, in the phytopathogenic fungus Bipolaris oryzae. Curr. Microbiol. 2008, 56, 115-121. [CrossRef] [PubMed]

7. Ballario, P.; Vittorioso, P.; Magrelli, A.; Talora, C.; Cabibbo, A.; Macino, G. White collar-1, a central regulator of blue light responses in Neurospora, is a zinc finger protein. EMBO J. 1996, 15, 1650-1657. [PubMed]

8. Schwerdtfeger, C.; Linden, H. Localization and light-dependent phosphorylation of white collar 1 and 2, the two central components of blue light signaling in Neurospora crassa. Eur. J. Biochem. 2000, 267, 414-422. [CrossRef] [PubMed]

9. Casas-Flores, S.; Rios-Momberg, M.; Bibbins, M.; Ponce-Noyola, P.; Herrera-Estrella, A. BLR-1 and BLR-2, key regulatory elements of photoconidiation and mycelial growth in Trichoderma atroviride. Microbiology 2004, 150, 3561-3569. [CrossRef] [PubMed]

10. Proietto, M.; Bianchi, M.M.; Ballario, P.; Brenna, A. Epigenetic and Posttranslational Modifications in Light Signal Transduction and the Circadian Clock in Neurospora crassa. Int. J. Mol. Sci. 2015, 16, 15347-15383. [CrossRef] [PubMed]

11. Hedtke, M.; Rauscher, S.; Röhrig, J.; Rodríguez-Romero, J.; Yu, Z.; Fischer, R. Light-dependent gene activation in Aspergillus nidulans is strictly dependent on phytochrome and involves the interplay of phytochrome and white collar-regulated histone H3 acetylation. Mol. Microbiol. 2015, 97, 733-745. [CrossRef] [PubMed]

12. Yu, Z.; Armant, O.; Fischer, R. Fungi use the SakA (HogA) pathway for phytochrome-dependent light signalling. Nat. Microbiol. 2016, 1, 16019. [CrossRef] [PubMed]

13. Fuller, K.K.; Ringelberg, C.S.; Loros, J.J.; Dunlap, J.C. The fungal pathogen Aspergillus fumigatus regulates growth, metabolism, and stress resistance in response to light. MBio 2013, 4, e00142-13. [CrossRef] [PubMed]

14. Hatakeyama, R.; Nakahama, T.; Higuchi, Y.; Kitamoto, K. Light represses conidiation in koji mold Aspergillus oryzae. Biosci. Biotechnol. Biochem. 2007, 71, 1844-1849. [CrossRef] [PubMed]

15. Schmidt-Heydt, M.; Rüfer, C.; Raupp, F.; Bruchmann, A.; Perrone, G.; Geisen, R. Influence of light on food relevant fungi with emphasis on ochratoxin producing species. Int. J. Food Microbiol. 2011, 145, $229-237$. [CrossRef] [PubMed]

16. Cheong, K.K.; Strub, C.; Montet, D.; Durand, N.; Alter, P.; Meile, J.C.; Schorr Galindo, S.; Fontana, A. Effect of different light wavelengths on the growth and ochratoxin A production in Aspergillus carbonarius and Aspergillus westerdijkiae. Fungal Biol. 2016, 120, 745-751. [CrossRef] [PubMed]

17. Alshannaq, A.; Yu, J.H. Occurrence, Toxicity, and Analysis of Major Mycotoxins in Food. Int. J. Environ. Res. Public Health 2017, 14, 632. [CrossRef] [PubMed]

18. Wild, C.P.; Miller, J.D.; Groopman, J.D. Mycotoxin control in low- and middle-income countries. In IARC Working Group Reports; Wild, C.P., Miller, J.D., Groopman, J.D., Eds.; International Agency for Research on Cancer: Lyon, France, 2015; Volume 9, pp. 1-54. ISBN 978-92-832-2510-2.

19. Umesha, S.; Manukumar, H.M.; Chandrasekhar, B.; Shivakumara, P.; Shiva Kumar, J.; Raghava, S.; Avinash, P.; Shirin, M.; Bharathi, T.R.; Rajini, S.B.; et al. Aflatoxins and food pathogens: Impact of biologically active aflatoxins and their control strategies. J. Sci. Food Agric. 2017, 97, 1698-1707. [CrossRef] [PubMed]

20. Vazquez, M.; Cepeda, A.; Prognon, P.; Mahuzier, G.; Blais, J. Cyclodextrins as modifiers of the luminescence characteristics of aflatoxins. Anal. Chim. Acta 1991, 255, 343-350. [CrossRef]

21. Suzuki, T.; Iwahashi, Y. Addition of Carbon to the Culture Medium Improves the Detection Efficiency of Aflatoxin Synthetic Fungi. Toxins (Basel) 2016, 8, 338. [CrossRef] [PubMed]

22. Maragos, C.M.; Appell, M.; Lippolis, V.; Visconti, A.; Catucci, L.; Pascale, M. Use of cyclodextrins as modifiers of fluorescence in the detection of mycotoxins. Food Addit. Contam. 2008, 25, 164-171. [CrossRef] [PubMed]

23. Yabe, K.; Nakamura, H.; Ando, Y.; Terakado, N.; Nakajima, H.; Hamasaki, T. Isolation and characterization of Aspergillus parasiticus mutants with impaired aflatoxin production by a novel tip culture method. Appl. Environ. Microbiol. 1988, 54, 2096-2100. [PubMed] 
24. Ramírez-Galicia, G.; Garduño-Juárez, R.; Gabriela Vargas, M. Effect of water molecules on the fluorescence enhancement of Aflatoxin B1 mediated by Aflatoxin B1:beta-cyclodextrin complexes. A theoretical study. Photochem. Photobiol. Sci. 2007, 6, 110-118. [CrossRef] [PubMed]

25. Degola, F.; Dall'Asta, C.; Restivo, F.M. Development of a simple and high-throughput method for detecting aflatoxins production in culture media. Lett. Appl. Microbiol. 2012, 55, 82-89. [CrossRef] [PubMed]

26. Yang, K.; Liang, L.; Ran, F.; Liu, Y.; Li, Z.; Lan, H.; Gao, P.; Zhuang, Z.; Zhang, F.; Nie, X.; et al. The DmtA methyltransferase contributes to Aspergillus flavus conidiation, sclerotial production, aflatoxin biosynthesis and virulence. Sci. Rep. 2016, 6, 23259. [CrossRef] [PubMed]

27. ImageJ. Available online: https:/ /imagej.nih.gov/ij/ (accessed on 9 August 2009).

28. Ministry of Agriculture, Forestry and Fisheries. Available online: http://www.maff.go.jp/j/seisan/boeki/ beibaku_anzen/kabikabidokukensa_surveillance/pdf/110816-3af.pdf (accessed on 8 June 2016).

2018 by the author. Licensee MDPI, Basel, Switzerland. This article is an open access article distributed under the terms and conditions of the Creative Commons Attribution (CC BY) license (http:/ / creativecommons.org/licenses/by/4.0/). 